\title{
Expression of p53 Protein in Prostate Carcinoma and its Correlation with Gleason Grade
}

\author{
Zakia Wahid ${ }^{1}$, Rozina Jaffer ${ }^{2}$, Sobia Khalid ${ }^{3}$, Haseeb Ahmed Khan ${ }^{1}$ and Sabiha Riaz ${ }^{1}$ \\ ${ }^{1}$ Department of Pathology, Fatima Memorial Hospital, Shadman, Lahore, Pakistan \\ ${ }^{2}$ Department of Pathology, Rahber Medical College, Lahore, Pakistan \\ ${ }^{3}$ Department of Pathology, University of Health Sciences, Lahore, Pakistan
}

\begin{abstract}
Objectives: To determine the correlation of p53 expression with Gleason score in prostate cancer.

Study Design: Descriptive study.

Place and Duration of Study: Department of Pathology, Fatima Memorial Hospital, Lahore; from January 2014 to August 2016.

Methodology: Thirty-seven samples of prostate carcinoma were graded using Gleason grading system. p53 expression was detected through routine immunohistochemical staining protocols and assessed semi-quantitatively using four-point scale score. The collected data was analysed statistically using Pearson's correlation coefficient.

Results: Out of a sample of 37, high grade tumours were observed in 21 (57\%) cases, making them the most prevalent type; while, p53 positive nuclear staining was observed in $26(70 \%)$ cases. Within the high grade tumours, strong positive p53 expression was observed in $8(38 \%)$ tumours; while, overexpression of p53 protein was seen in 7 (33\%) cases. In contrast, overexpression of p53 was absent in low grade tumours.
\end{abstract}

Conclusion: A statistically significant correlation was observed between p53 positive expression and high Gleason grade.

Key Words: Prostate cancer, Immunohistochemistry, p53 protein, Gleason score.

How to cite this article: Wahid Z, Jaffer R, Khalid S, Khan HA, Riaz S. Expression of p53 Protein in Prostate Carcinoma and its Correlation with Gleason Grade. J Coll Physicians Surg Pak 2020; 30(06):643-645 https://doi.org/10.29271/jcpsp.2020.06.643.

\section{INTRODUCTION}

There is evidence to suggest that prostate carcinoma is the second most prevalent malignancy in males the world over. ${ }^{1}$ It is considered the third most common malignancy among males in Pakistan, effecting around $3.8 \%$ of male population. ${ }^{2} \mathrm{~A}$ major challenge in its treatment is the current inability to readily distinguish between indolent and aggressive cancers. Therefore, identification of biomarkers that differentiate these two types can greatly assist in diagnosis and better management. Tumor suppressor genep 53 is one of such biomarkers. ${ }^{3}$ Various studies among western population have indicated that low grade prostate cancers show low p53 expression; while, high or overexpression of p53 protein is seen in highergrades of prostate cancers. ${ }^{4}$

Correspondence to: Dr. Zakia Wahid, Department of Pathology, Fatima Memorial Hospital, Shadman, Lahore, Pakistan

E-mail: zakiasaif@yahoo.com

Received: October 24, 2018; Revised: October 03, 2019;

Accepted: December 11, 2019

DOI: https://doi.org/10.29271/jcpsp.2020.06.643
This study examines the existence of such relationship in prostatic samples analysed in a tertiary healthcare centre in Lahore. The objective of the study was to determine the correlation of p53 expression with Gleason score in prostate cancer.

\section{METHODOLOGY}

This study was conducted at the Department of Pathology, Fatima Memorial Hospital, Lahore. It included all transurethrally resected biopsy specimen of primary prostate carcinoma received from January 2014 to August 2016 . With $90 \%$ power of the study and $5 \%$ level of significance, a sample size of 37 was determined. The samples were selected using consecutive nonprobability sampling method.

Standard haematoxylin and eosin staining method was applied on formalin fixed and paraffin embedded tissue sections. Tumours were histologically graded using Gleason's grading system. Those obtaining Gleason score of 6 or less, 7 and 8-10 were respectively considered as low, intermediate and high gradetumours. ${ }^{1}$

Routine immunohistochemistry was used to detect $\mathrm{p} 53$ protein expression. Both positive and negative controls were run concurrently. Dark brown nuclear immunoreactivity was taken as positive staining and assessed using four-point scale score. The results were interpreted as positive for any number of 
nuclear staining. 0 score was assigned when no staining was observed. Samples with p53 score of 1 (1-33\%) were classified as low-staining, samples with p53 score of 2 (34-67\%) were classified as high-staining group; while, samples with p53 score of 3 (68-100\%) were classified as group with overexpression. ${ }^{5}$

The results were statistically analysed by using the Pearson's correlation coefficient on STATA statistical software. $P<0.05$ was taken as significant. Frequency with percentages for categorical variable was calculated.

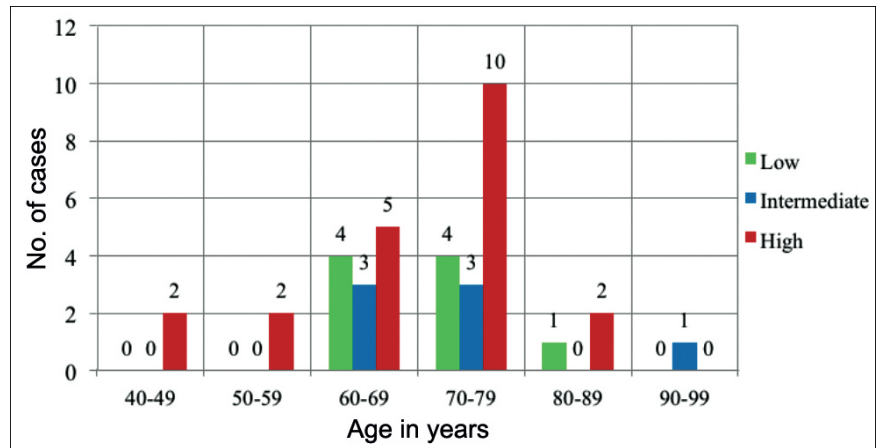

Figure 1: Age distribution in different histological grades of prostatic adenocarcinoma.

\section{RESULTS}

Table I shows that out of 37 sample tumors, 21 (57\%) obtained a Gleason score of 8-10; while, 9 (24\%) and 7 (19\%) obtained Gleason score of 7 and 2-6, respectively. Thus high grade prostate carcinoma is the most prevalent type, almost twice more prevalent than the low grade and thrice more prevalent than the intermediate grade prostate carcinoma.

Positive p53 expression was observed in almost two-thirds ( 26 or $70 \%$ ) samples. Within the samples with positive 053 expression, high $(2+)$ and overexpression $(3+)$ was observed in 20 $(54 \%)$; while, low expression (1+) was observed in 6 (16\%). Further, considerably high staining scores were observed in samples with high and intermediate grade carcinomas. As indicated by Table 2 , out of 8 samples with p53 staining score of $3+, 7(88 \%)$ were high grade while only 1 (12\%) was of intermediate grade. Similarly, out of 12 samples with p53 staining score of $2+, 8(66 \%)$ were of high-grade while $2(17 \%)$ each were of intermediate and low grade. Maximum number of low grade carcinoma were observed with p53 staining score of $1+$. Out of 6 samples with this staining score, 2 (34\%) were of high and intermediate grade while 4 or $66 \%$ were of low-grade. As far as samples with p53 negative expression, no significant difference in distribution of grades was observed. Out of 11 samples in this category, 5 (45\%) were of high-grade while 3 ( $27 \%$ ) each were of intermediate and low grade.

Correlation between p53 protein expression and Gleason score was worked out using Pearson correlation coefficient. The correlation was observed to be positive $(r=0.4137$, $p=0.019)$ for the entire sample $(n=37)$ and strongly positive $(r=0.5832, p=0.0018)$ for samples with positive $p 53$ expres$\operatorname{sion}(n=26)$.

\section{DISCUSSION}

Research points out that in contrast to the developed economies, majority of patients of prostate cancer in developing economies present with high grade and advance stage. . $^{6-8}$ As indicated in Figure1, over half of the studied patients (57\%) were diagnosed with high Gleason grade tumor. The findings of present study are in accordance with aforesaid research albeit ingradeonly.

Prostate cancer may exhibit rapid aggressive or an indolent slow growth. Thus, under or over diagnosis of the tumor can lead to maltreatment. ${ }^{9}$ Established limitations of morphological estimation dictate the need for auxiliary studies to further refine diagnosis and management plans. Out of the many possible areas, study of p53 gene is gaining prominence as it is the primary regulator of apoptotic pathway. Mutational change and deletion causes loss of p53 functions which can lead to inappropriate cell growth, increased survival and genomic instability that ultimately leads to tumor-genesis. ${ }^{10,11}$ p53 gene mutations are associated with poor prognosis in several human malignancies including prostate cancer. ${ }^{12}$ In this study, an elaborate p53 protein expression was detected in $70 \%$ (26) samples which closely matches with that reported by Verma et al. ${ }^{4}$ However, it may be noted that various studies have reported presence of p53 immuno-expression between $4 \%$ to $79 \%$. 4,15 These variations are attributable to methodological differences in tissue sampling, scoring and the antibody clone used.

Several studies also point out a positive correlation among stronger positive expression of p53 protein and higher grade of prostate cancer. ${ }^{4,13}$ In this study, most of the tumours with low Gleason grade exhibited lower p53 positive expression in comparison to the tumours scoring intermediate and high Gleason grades. However, the incidence of highand overexpression of p53 protein was much higher in tumours with intermediate and high grades as compared to tumours with low Gleason grades. To sum up, a positive and statistically significant correlation was noticed between p53 immunohistochemical expression and high Gleason grade $(p=0.0018)$. This finding is consistent with various other studies. ${ }^{4,13,14}$

The latent significance of p53 overexpression as a prognostic indicator has been examined in a large number of carcinomas. Saidi et al. noted significantly worse prognosis in patients with high expression ofp53asagainstpatients with negative expression. ${ }^{11}$ Similarly, Kaur et al. and Kluth et al. confirmed that in case of patients treated with radical prostatectomy for localised prostate cancer, p53 protein expression stands out as an independent prognostic biomarker for disease-free survival. ${ }^{14,15}$

This study suggests that p53 protein is a good surrogate biomarker for assessing malignant potential of a tumor and disease prognosis in local population. Besides assisting clinicians in selection of the patients for adjuvant and neo-adjuvant therapy, it can also enhance and augment their ability to counsel individual patients forchoice of treatment. 
Table I: p53 expression and Gleason score.

\begin{tabular}{|c|c|c|c|c|c|c|}
\hline \multirow{2}{*}{ Gleason score } & \multirow{2}{*}{ Frequency } & \multicolumn{5}{|c|}{ p53 staining expression } \\
\hline & & -ve* & +ve* & Low* & High* & Over expression* \\
\hline Low grade $(2-6)$ & $9(24 \%)$ & $3(33 \%)$ & $6(66 \%)$ & $4(44 \%)$ & $2(23 \%)$ & $0(0 \%)$ \\
\hline Intermediate grade (7) & $7(19 \%)$ & $3(43 \%)$ & $4(57 \%)$ & $1(14 \%)$ & $2(28 \%)$ & $1(14 \%)$ \\
\hline High grade $(8-10)$ & $21(57 \%)$ & $5(24 \%)$ & $16(76 \%)$ & $1(5 \%)$ & $8(38 \%)$ & $7(33 \%)$ \\
\hline Total & $37(100 \%)$ & $11(30 \%)$ & $26(70 \%)$ & $6(16 \%)$ & $12(32 \%)$ & $8(22 \%)$ \\
\hline
\end{tabular}

Table II: Positive p53 expression and Gleason score.

\begin{tabular}{|c|c|c|c|c|}
\hline \multirow{2}{*}{ Gleason score } & \multirow{2}{*}{ Frequency } & \multicolumn{3}{|c|}{ p53 staining expression } \\
\hline & & Low & High & Over expression \\
\hline Low grade (2-6) & 6 & $4(66 \%)$ & $2(34 \%)$ & $0(0 \%)$ \\
\hline Intermediate grade (7) & 4 & $1(25 \%)$ & $2(50 \%)$ & $1(25 \%)$ \\
\hline High grade $(8-10)$ & 16 & $1(6 \%)$ & $8(50 \%)$ & $7(44 \%)$ \\
\hline Total & 26 & $6(23 \%)$ & $12(46 \%)$ & $8(31 \%)$ \\
\hline
\end{tabular}

\section{CONCLUSION}

A statistically significant correlation exists between p53 positive expression and Gleason score. Moreover, overexpression of p53 protein was absent in low Gleason score tumors; and high and overexpression of p53 protein was seen in intermediate and high grade tumors.

\section{ETHICAL APPROVAL:}

The approval of the study was taken from the Institutional Review Board of FMH College of Medicine \& Dentistry before commencing the research (IRB \# FMS-4-2016-IRB-M-139 already submitted).

\section{PATIENTS CONSENT:}

The informed consents were obtained from all patients for the publication of this study.

\section{CONFLICT OF INTEREST:}

The authors declare that they have no competing interests.

\section{AUTHORS' CONTRIBUTION:}

ZW: Collected samples and patients related information, carried out histopathological analysis, literature review and wrote the primary draft.

SK, RJ: Worked out study design, interpretation of data.

HAK: Critically reviewed the manuscript for consistency and correctness. Also helped the whole team in developing sound and logical conclusions.

SR: Provided a critical insight into all aspects of the study and also repeatedly reviewed the manuscript to bring it into a publishable shape.

\section{REFERENCES}

1. Ferlay J, Soerjomataram I, Dikshit R, Eser S, Mathers C, Rebelo $\mathrm{M}$, et al. Cancer incidence and mortality worldwide: Sources, methods and major patterns in globocan 2012. Int J Cancer 2015; 136(5):E359-86.

2. Aziz Z, Sana S, Saeed S, Akram M. Institution-based tumor registry from Punjab: Five year data based analysis. J Med Pak Assoc 2003; 53(8):350-3.

3. Paker SK, Kilicarslan B, Ýftcýoglu AM, Oztekin S, Sargin FC,
Erdogru $\mathrm{T}$, et al. Relationship between apoptosis regulator proteins (bcl-2 and p53) and Gleason score in prostate cancer. Pathol\& Oncol 2001; 7(3):209-12.

4. Verma R, Gupta V, Singh J, Verma M, Gupta G, Gupta S, et al. Significance of p53 and ki-67 expression in prostate cancer. Urol Ann 2015; 7(4):488.

5. Munda M, Hajdinjak T, Kavalar R, Martincic DS. p53, Bcl-2 and AgNOR tissue markers: Model approach in predicting prostate cancer characteristics. J Int Med Res 2009; 37(6):1868-76.

6. Cooperberg MR, Broering JM, Carroll PR. Time trends and local variation in primary treatment of localized prostate cancer. J Clin Oncol 2010; 28(7):1117.

7. Siegel RL, Miller KD, Jemal A. Cancer statistics, 2015. CA Cancer J Clin 2015; 65(1):5-29.

8. Kehinde EO, Maghrebi M, Anim JT. The importance of determining the aggressiveness of prostate cancer using serum and tissue molecular markers. Can J Urol 2008; 15(2):3967-74.

9. Ross JS, Sheehan CE, Dolem EM, et al. Morphologic and molecular prognostic markers in prostate cancer. Adv Anat Pathol 2007; 9(2):115-28.

10. Goh AM, Coffill CR, Lane DP. The role of mutant $\mathrm{p} 53$ in human cancer. J pathol 2011; 223(2):116-26.

11. Saidi S, Georgiev V, Stavridis S, Penev M, Stankov O, Dohcev S, et al. Evaluation of the value of p53 protein expression in the extra-capsular extension of prostate cancer. Prilozi 2011; 32(2):213-20.

12. Navone NM, Troncoso P, Pisters LL, Goodrow TL, Palmer JL, et al. p53 protein accumulation and gene mutation in the progression of human prostate carcinoma. J Nati Cancer Inst 1993; 85(20):1657-69.

13. Brosh R, Rotter V. When mutants gain new powers: News from the mutant p53 field. Nat Rev Cancer 2009; 9(10): 701.

14. Kaur H, Paul M, Manjari M, Sharma S, Bhasin TS, Mannan R. Ki-67 and p53 immunohistochemical expression in prostate carcinoma: An experience from a tertiary care centre of North India. Ann Pathol Lab Med 2016; 3(6):A509-16.

15. Kluth M, Harasimowicz S, Burkhardt L, Grupp K, Krohn A. Clinical significance of different types of p53 gene alteration in surgically treated prostate cancer. Int J Cancer 2014; 135(6): 1369-80. 